Louisiana State University

LSU Digital Commons

Faculty Publications

School of Animal Sciences

$1-1-2014$

\title{
Differential effects of Akt isoforms on somatic cell reprogramming
}

\author{
Yong Tang \\ University of Connecticut \\ Zongliang Jiang \\ University of Connecticut \\ Yan Luo \\ University of Connecticut \\ Xueming Zhao \\ University of Connecticut \\ Ling Wang \\ University of Connecticut
}

See next page for additional authors

Follow this and additional works at: https://digitalcommons.Isu.edu/animalsciences_pubs

\section{Recommended Citation}

Tang, Y., Jiang, Z., Luo, Y., Zhao, X., Wang, L., Norris, C., \& Tian, X. (2014). Differential effects of Akt isoforms on somatic cell reprogramming. Journal of Cell Science, 127 (18), 3998-4008. https://doi.org/ $10.1242 / j c s .150029$

This Article is brought to you for free and open access by the School of Animal Sciences at LSU Digital Commons. It has been accepted for inclusion in Faculty Publications by an authorized administrator of LSU Digital Commons.

For more information, please contact ir@lsu.edu. 


\section{Authors}

Yong Tang, Zongliang Jiang, Yan Luo, Xueming Zhao, Ling Wang, Carol Norris, and Xiuchun Cindy Tian 


\title{
RESEARCH ARTICLE
}

\section{Differential effects of Akt isoforms on somatic cell reprogramming}

\author{
Yong Tang ${ }^{1}$, Zongliang Jiang ${ }^{1}$, Yan Luo ${ }^{1}$, Xueming Zhao ${ }^{1}$, Ling Wang ${ }^{1}$, Carol Norris ${ }^{2}$ and Xiuchun (Cindy) Tian ${ }^{1, *}$
}

\begin{abstract}
Akt plays an important role in cell growth, proliferation and survival. The specific roles of the three Akt isoforms in somatic cell reprogramming have not been investigated. Here we report that, during iPSC generation, enhanced Akt1 activity promotes complete reprogramming mainly through increased activation of Stat3 in concert with leukemia inhibitory factor (LIF) and, to a lesser extent, through promotion of colony formation. Akt1 augments Stat3 activity through activation of mTOR and upregulation of LIF receptor expression. Similarly, enhanced Akt2 or Akt3 activation also promotes reprogramming and coordinates with LIF to activate Stat3. Blocking Akt1 or Akt3 but not Akt2 expression prohibits cell proliferation and reprogramming. Furthermore, the halt in cell proliferation and reprogramming caused by mTOR and Akt inhibitors can be reversed by inhibition of GSK3. Finally, we found that expressing the GSK3 $\beta$ target Esrrb overrides inhibition of Akt and restores reprogramming. Our data demonstrated that during reprogramming, Akt promotes establishment of pluripotency through co-stimulation of Stat3 activity with LIF. Akt1 and Akt3 are essential for the proliferation of reprogrammed cells, and Esrrb supports cell proliferation and complete reprogramming during Akt signaling.
\end{abstract}

KEY WORDS: Akt, GSK3, Isoform, Reprogramming, Stat3, iPSC

\section{INTRODUCTION}

The phosphatidylinositol 3-kinase (PI3K)/Akt (PI3K/Akt) pathway has important functions in normal cell and cancer cell development (Manning and Cantley, 2007; Vanhaesebroeck and Alessi, 2000). Activated by receptor tyrosine kinases, PI3K converts phosphatidylinositol (4,5)-bisphosphate (PIP2) to phosphatidylinositol $(3,4,5)$-bisphosphate (PIP3), which recruits Akt to the plasma membrane, where Akt is activated by both phosphoinositide-dependent kinase 1 (PDK1) and mammalian target of rapamycin complex 2 (mTORC2) (Alessi et al., 1997; Sarbassov et al., 2005). Activated Akt regulates many downstream signaling pathways, such as mammalian target of rapamycin complex 1 (mTORC1), glycogen synthase kinase 3 (GSK3) and forkhead box O transcription factors (FOXO). These pathways control diverse functions including cell growth, proliferation, survival and metabolism (Manning and Cantley, 2007; Vanhaesebroeck and Alessi, 2000).

\footnotetext{
${ }^{1}$ Center for Regenerative Biology, Department of Animal Science, University of Connecticut, Storrs, CT 06269, USA. ${ }^{2}$ Department of Molecular and Cellular Biology/Biotechnology and Bioservices Center, University of Connecticut, Storrs, CT 06269, USA.

*Author for correspondence (xiuchun.tian@uconn.edu)
}

Received 23 January 2014; Accepted 7 July 2014
There are currently three known members of the Akt protein family, namely Akt1, Akt2 and Akt3, each of which is encoded by a different gene. These isoforms share a similar N-terminal Pleckstrin-homology domain and a central serine-threonine kinase domain, and their amino acid sequences are highly conserved from mice to humans (Yang et al., 2004). Work with knockout mouse models has shown a role for Akt1 in organismal growth and cell survival (Chen et al., 2001; Cho et al., 2001b), for Akt2 in growth regulation and glucose metabolism (Cho et al., 2001a; Garofalo et al., 2003), and for Akt3 in post-natal brain development (Tschopp et al., 2005). However, there are also functional overlaps between the three isoforms. Of note, isoformspecific Akt deregulation is frequently observed in several cancers, including amplification of Aktl in thyroid and nonsmall cell lung cancers (Lee et al., 2011; Saji et al., 2011), of Akt2 in glioma (Zhang et al., 2009), and of Akt3 in melanoma (Stahl et al., 2004). Despite many studies, the pathways through which specific isoforms of Akt exert their effects still remain largely undefined (Chin and Toker, 2011).

Induced pluripotent stem cell (iPSC) technology (Takahashi and Yamanaka, 2006) holds great potential for regenerative medicine, by generating embryonic stem cell (ESC)-like cells directly from somatic cells. Additionally, the short duration of iPSC generation (2-3 weeks) provides a convenient way to study mechanisms of cellular dedifferentiation, a process also associated with tumor development and metastasis (Gabbert et al., 1985; Herreros-Villanueva et al., 2013; Schwitalla et al., 2013; Zhang et al., 2013). iPSCs can be induced by ectopic expression of different sets of transcription factors, e.g. Oct4, Klf4, Sox2 and Myc (OKSM) (Takahashi and Yamanaka, 2006) or Oct4, Sox2, Nanog and Lin28 (Yu et al., 2007). In mice, the activation of Janus-associated kinase (Jak)/signal transducer and activator of transcription 3 (Stat3) is required for the establishment of pluripotency during somatic cell reprogramming (Tang et al., 2012; van Oosten et al., 2012). The Jak/Stat3 pathway is activated by leukemia inhibitory factor (LIF), a cytokine required for the self-renewal of ESCs (Niwa et al., 1998; Smith et al., 1988; Williams et al., 1988). In ESCs, LIF also activates both the PI3K/Akt and extracellular-signal-regulated kinase 1/2 (Erk1/2) pathways (Burdon et al., 2002; Niwa et al., 2009; Paling et al., 2004). Erk1/2 activity signals differentiation in ESCs; this activity appears to act as a counter-balance to the self-renewal signal provided by STAT3 (Burdon et al., 2002; Burdon et al., 1999). Although Akt1 activation was shown to have paradoxical roles in reprogramming mediated by cell fusion or nuclear transfer (Nakamura et al., 2008), forced Akt1 activation was reported to sustain mouse ESC self-renewal in the absence of LIF (Watanabe et al., 2006) and promote pluripotent stem cell derivation from mouse primordial germ cells (Kimura et al., 2008). Akt also maintains pluripotency of human ESCs by modulating activity of Smad2 and Smad3 (Singh et al., 2012). However, the exact roles of the different Akt isoforms during iPSC generation are still unknown. 
By using mouse embryonic fibroblasts (MEFs) harboring the green fluorescence protein (GFP) expression cassette driven by the Oct4-promoter (OG-MEF), we investigated the roles of Akt1, Akt2 and Akt3 in mouse iPSC generation through both gain- and loss-of-function studies. Our findings revealed the common and isoform-specific roles of Akt1, Akt2 and Akt3 during the reprogramming process, and a key role of glycogen synthase kinase-3 beta (GSK3 $\beta$ )/estrogen-related receptor beta (Esrrb) axis in reprogramming.

\section{RESULTS \\ Enhanced Akt1 activity promotes somatic cell reprogramming}

We first asked whether enhanced Akt1 activity could improve direct reprogramming of MEFs into iPSCs. Using a pMXsretroviral vector, we constructed a myristoylated, constitutively active Akt1 (CA-Akt1) (Nesterov et al., 2001; Watanabe et al., 2006). Male OG-MEFs reprogrammed with OKSM (day 0) were transduced with either CA-Akt1 or vector control on day 2 and cultured with or without LIF. Dome-shaped, ESC-like colonies started to appear 1 week after OKSM transduction in all treatment groups (data not shown). By day 11, Oct4-GFP fluorescence could be observed in CA-Akt1 transduced cells cultured with LIF, but not in the vector controls with or without LIF (Fig. 1A). Two and three weeks after OKSM induction, cells expressing CA-Akt1 showed increased GFP-positive colony formation compared to controls (Fig. 1A,B); the number of GFP-positive colonies was further augmented in the presence of LIF $(\sim 2.8$-fold higher than control at 3 weeks, Fig. 1B). Addition of LIF to the vector controls had no effect on GFP-positive colony formation at 3 weeks (Fig. 1B), which is likely to be an effect of LIF paracrine signaling by the MEF feeder cells (Rathjen et al., 1990; Stewart et al., 1992), and is similar to what we have observed previously (Tang et al., 2012). Furthermore, CA-Akt1 plus OKSM not only mildly increased total ESC-like colony formation (Table 1), but also significantly enhanced the proportion of GFP-positive colonies as a percentage of total colonies (Fig. 1C). When used in combination with LIF, the effect of CA-Akt1 on improving GFP-positive colony ratio was statically significant $(74 \%)$ compared to controls that had been treated with or without LIF (38 or $41 \%$ ) (Fig. 1C). Therefore, constitutively active Akt1 improves reprogramming by increasing both the total number of colonies formed and the percentage of pluripotent (GFP-positive) colonies. This latter effect is reminiscent of the function of Stat3 in somatic cell reprogramming that we described previously (Tang et al., 2012).

We then performed additional characterizations to confirm that the GFP-positive colonies were, indeed, pluripotent. We found that the CA-Akt1/OKSM-induced GFP-positive colonies shared similar properties with those of standard ESCs (R1), including the ability to form tight, compact colonies with strong alkaline phosphatase (ALP) activity (Fig. 1D). They readily propagated in 2i/LIF medium, the selective medium for ground state pluripotent ESCs (Silva et al., 2009; Ying et al., 2008). qRT-PCR and immunostaining revealed that these colonies expressed pluripotent genes, and surface markers including Oct4, Sox2, Nanog and SSEA-1 at either greater or comparable levels to those of ESCs (Fig. 1E; supplementary material Fig. S1-S3), and showed silenced retroviral Akt1 transgene expression in induced colonies (supplementary material Fig. S4). They efficiently differentiated into EBs in vitro (Fig. 1F), and developed into cells expressing markers of the three germ layers (Fig. 1G; supplementary material Fig. S4). Moreover, these cells also formed beating myocardiocytes upon differentiation (supplementary material Movie 1). Taken together, these results demonstrate that enhanced Akt1 activation significantly improves the efficiency of iPSC generation by OKSM.

\section{Akt1 enhances LIF-stimulated Stat3 activation and complete reprogramming}

We then aimed to determine the mechanism by which Akt1 activity promotes reprogramming. It has been shown that mTOR directly phosphorylates Ser727 of Stat3 to promote Stat 3 activation, as indicated by the increased phosphorylation of Tyr705 (Yokogami et al., 2000). Expression of an oncogenic Akt1 mutant or activation of mTORC1 in MEFs resulted in activation of the Stat3/p63/Notch cascade (Ma et al., 2010), indicating that the Akt/mTOR axis triggers Stat3 activation, although it is unclear whether enhanced activation of wild-type Akt will also stimulate Stat3. The collaborative effect we observed between CA-Akt1 and LIF in promoting the establishment of pluripotency during OKSM reprogramming (Fig. 1B,C), led us to investigate a possible activation of the Stat3 pathway by Akt1. Western blot analysis revealed that CA-Akt1-transduced MEFs exhibited increased levels of phosphorylated Stat3 (pStat3) at Tyr705 in the presence of LIF (Fig. 2A). In another batch of MEFs, blocking Akt activity in CA-Akt1-transduced MEFs by applying the specific allosteric inhibitor MK2206 (Hirai et al., 2010) resulted in a dramatic decrease in both phosphorylated Akt (pAkt) and LIF-induced phosphorylation of Stat3 (Fig. 2B,C). Similarly, blocking mTORC1 activity with a low dose of rapamycin (Heitman et al., 1991; Laplante and Sabatini, 2012) inhibited phosphorylation of Stat3 while exerting a minimal effect on pAkt levels (Fig. 2B,C). These results demonstrate that CA-Akt1 collaborates with LIF to maximize Stat3 activation through the Akt/mTORC1 axis.

To investigate other possible mechanisms by which Akt1 promotes Stat3 activation, we evaluated the expression level of LIF receptor (LIFR). qRT-PCR analysis showed that the CAAkt1 transduced MEFs expressed significantly higher levels of LIFR than controls with or without stimulation with LIF (Fig. 2D). This increase was accompanied by the increased expression of SOCS3, a direct target of Stat3, with the highest level of SOCS3 induced by CA-Akt1 plus LIF (Fig. 2D). Therefore, Akt1 not only directly enhances Stat3 phosphorylation through Akt/mTOR, but also exerts a positive feedback on LIFR expression, which further enhances the activities of Stat3 and Akt upon stimulation with LIF.

We have previously demonstrated that Stat3 activation is essential for the establishment of pluripotency in induced colonies during late-stage reprogramming (Tang et al., 2012). Results presented here show that enhanced Akt1 activity not only increases the levels of pStat3, but also increases the percentage of pluripotent (GFP-positive) colonies, an effect also shown by Stat3 itself. These data strongly indicate that Akt1 promotes reprogramming through maximizing Stat3 activity. We then asked whether Akt1 can establish pluripotency via a pathway independent of Stat3. We reprogrammed OG-MEFs by using OKSM together with LIF, CA-Akt1 and Jak inhibitor I (Jaki), which blocks Stat3 activation (Niwa et al., 2009; Tang et al., 2012). Inhibiting Stat3 activity blocked almost all GFP-positive colony formation, even in the presence of CA-Akt1 (Fig. 2E,F), while having no impact on total colony formation (Fig. 2F)

\section{(n)}


A

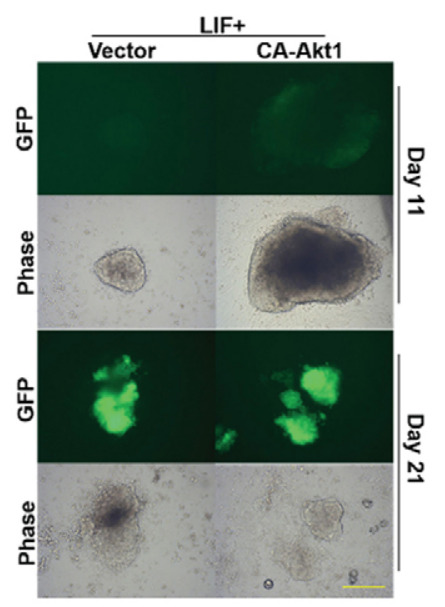

C

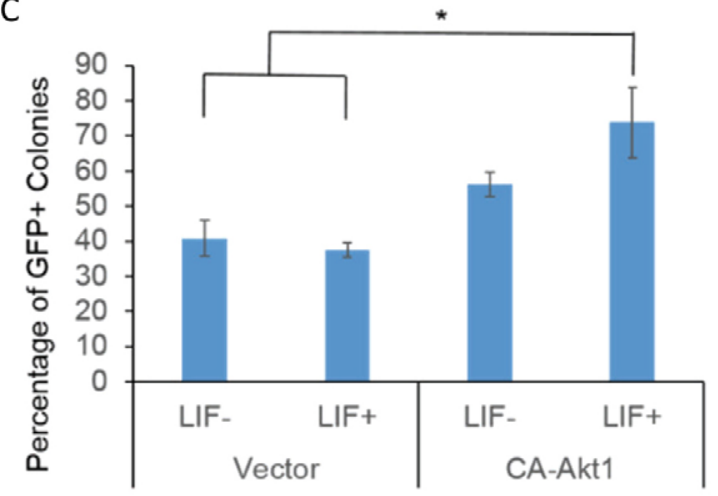

$E$

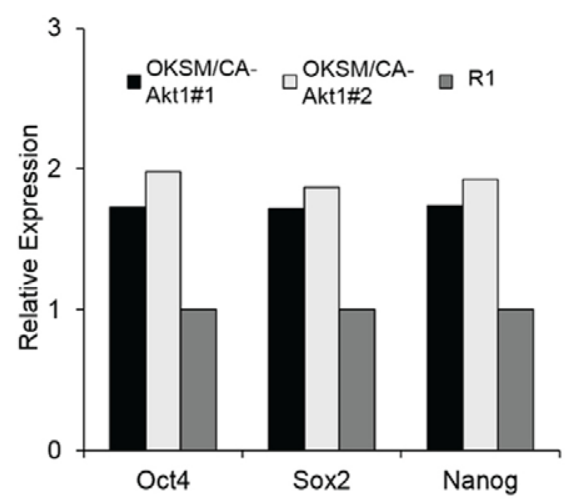

B

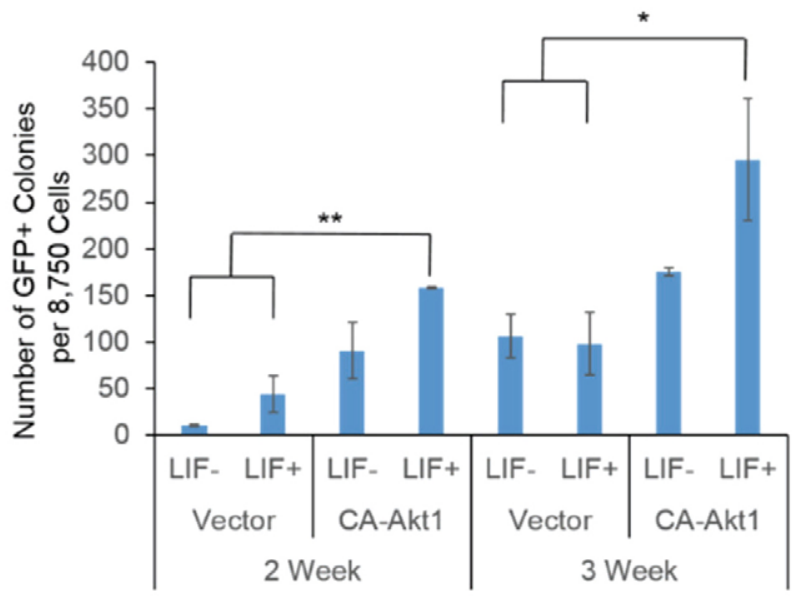

D
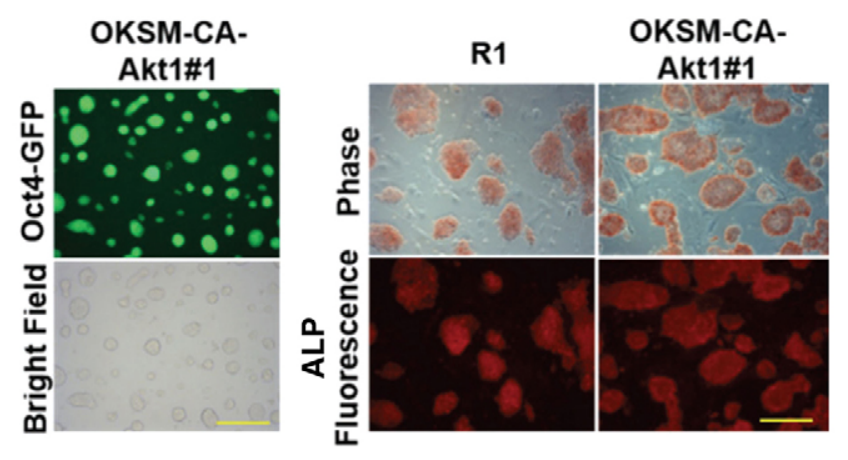

G

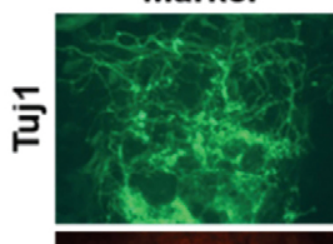

Marker

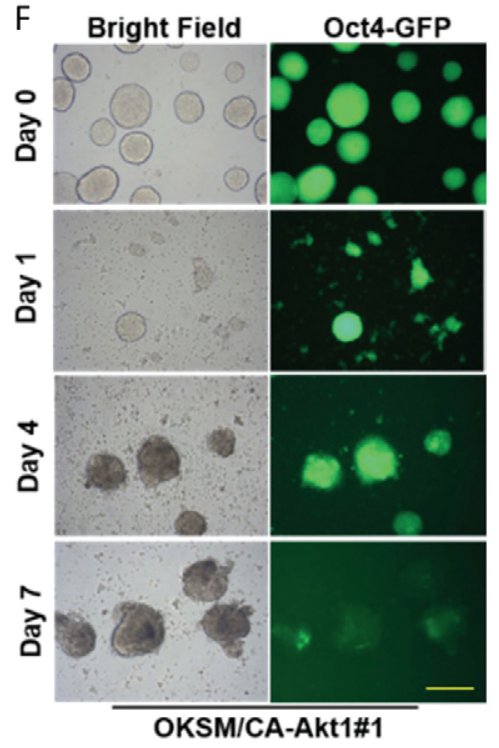

DAPI

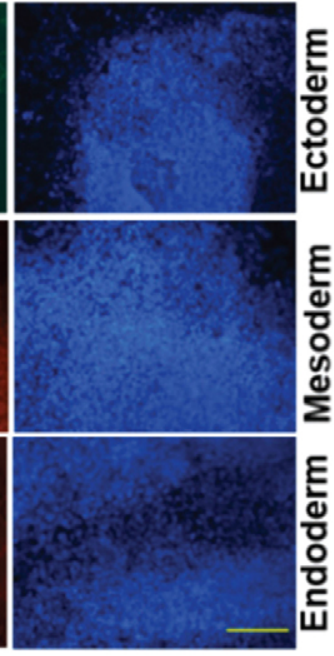

Fig. 1. See next page for legend.

(previously reported by Tang et al., 2012). Taken together, our data illustrate that enhanced Akt1 activation improves establishment of pluripotency in induced colonies through maximizing Stat3 activity in concert with LIF. This is achieved through direct phosphorylation of Stat3 by the Akt/mTOR axis and through promotion of LIFR expression.
Akt2 and Akt3 also promote reprogramming efficiency, and stimulate Stat3 activity

To investigate the possible roles of the two other Akt isoforms in reprogramming, we expressed myristoylated CA-Akt2 or CAAkt3 in retroviral pMCs-vectors and tested their effect on OKSMinduced reprogramming. We found that enhanced activities of 
Fig. 1. Enhanced Akt1 activation promotes MEF reprogramming (A) Representative colonies induced by transduction of OG-MEF with OKSM in combination with CA-Akt1 or vector control, followed by growth in LIFcontaining (LIF+) reprogramming medium for 11 or 21 days after the initial OKSM transduction. Expression of the Oct4-GFP-positive reporter indicates activation of the endogenous Oct4 gene (Scale bar: $250 \mu \mathrm{m}$ ). (B) Number of GFP-positive colonies at 2 or 3 weeks after transduction of OG-MEF with OKSM plus retroviral vector control or CA-Akt1, followed by growth in reprogramming medium with $(+)$ or without $(-)$ LIF. Activated Akt1 had a collaborative effect with LIF on the development of GFP-positive colonies. (C) GFP-positive colonies as a percentage of total ESC-like colonies induced by OKSM plus retroviral vector control or CA-Akt1. Colonies were counted 3 weeks after OKSM transduction. Together, stimulation with LIF and constitutively active Akt1 (CA-Akt1) increased the proportion of GFP-positive colonies during reprogramming. (D) GFP fluorescence and ALP staining of a representative iPSC line induced by OKSM and CA-Akt1 reprogramming and cultured in $2 \mathrm{i} / \mathrm{LIF}$-containing medium for five passages. ALP staining is also shown for a control ESC cell line (R1). (Scale bar: $610 \mu \mathrm{m})$. The iPSC colonies strongly expressed ALP. (E) Relative levels of mRNA for endogenous Oct4, Sox2 and Nanog in two representative iPSC lines at passage 6 . The values were normalized to the expression of GAPDH and expressed relative to the control ESC line R1. These iPSC colonies expressed endogenous pluripotent gene mRNAs at greater levels than ESCs. (F) Morphology and GFP expression in the original iPSC colonies (day 0 ) and 1, 4 or 7 days after transfer to EB differentiation conditions, showing gradually diminished Oct4-GFP expression during differentiation (Scale bar: $250 \mu \mathrm{m}$ ). The iPSC colonies differentiated efficiently into EBs. (G) Immunostaining for markers of ectoderm (Tuj1), mesoderm (Flk1) and endoderm (Sox17) at day 14 of EB differentiation. Nuclei of the cells were counterstained with DAPI (Scale bar: $120 \mu \mathrm{m}$ ). The iPSCs differentiated into cells expressing markers of all three germ layers.

Akt2 and Akt3 both significantly promoted reprogramming by increasing the number of GFP-positive colonies compared to control $(\sim 4.2 \times$ for Akt2 and $2.1 \times$ for Akt3, Fig. 3A). Also, as for Akt1, CA-Akt 2 or CA-Akt3 expression only slightly increased the total number of ESC-like, ALP-positive colonies (1.6-1.8 $\times$ ) during reprogramming (Fig. $3 \mathrm{~B}$ and Table 2).

We then evaluated whether Akt2 and Akt3 also stimulate Stat3 activity. Western blot analysis revealed that both CA-Akt2- and CA-Akt3-expressing MEFs showed enhanced levels of pStat3 when compared with controls following stimulation with LIF (Fig. 3C). These data strongly indicate that Akt2 and Akt3 also collaborate with LIF in maximizing Stat3 activation, and in promoting reprogramming efficiency.

\section{Akt1 and Akt3, but not Akt2, are essential for cell proliferation and reprogramming}

We further evaluated whether all Akt isoforms are essential for the reprogramming process. Using lentiviral short hairpin RNA (shRNA) constructs designed specifically against each Akt isoform (shAkt1, shAkt2, and shAkt3), we were able to knock down both Akt1 and Akt 2 mRNA levels in MEFs by more than 95\% (Fig. 3D). We used two constructs targeting different

Table 1. Number (mean \pm s.d.) of total ESC-like colonies induced by OKSM plus retroviral vector or CA-Akt1 in OGMEFs, 3 weeks after OKSM transduction. Starting cell number: $8750(n=2)$

\begin{tabular}{lll}
\hline & \multicolumn{2}{l}{ OKSM } \\
\cline { 2 - 3 } Total ESC-like colonies & Vector & CA-Akt1 \\
\hline LIF- & $236.3 \pm 42.9$ & $321.5 \pm 7.8$ \\
LIF+ & $252.7 \pm 61.3$ & $378.0 \pm 36.8$ \\
\hline
\end{tabular}

LIF-, cells cultured without LIF; LIF+, cells cultured with LIF.
mRNA regions of Akt3 to ensure its maximal knockdown (Fig. 3D). We then transduced OG-MEFs with Akt shRNA constructs 2 days after OKSM transduction. To avoid possible interference of lentiviral Akt knockdown on feeders, the cells were reprogrammed without a feeder layer. We found that knockdown of either Akt1 or Akt3 dramatically reduced both the GFP-positive and total colony formation (Fig. 3E,F). This is correlated with an inhibition of the growth and proliferation of OKSM transduced OG-MEFs by Akt1 or Akt3 knockdown (Fig. 3G). By contrast, knockdown of Akt2 had minimal impact on both total ALP- and GFP-positive colony formation (Fig. 3E,F), and did not affect the proliferation of OKSM transduced cells (Fig. 3G).

To find out whether the negligible effect of Akt2 knockdown on reprogramming is due to a much lower expression of Akt2 compared to the other two Akt isoforms, we evaluated the relative expression of the three Akt isoforms in MEFs. Interestingly, both Akt2 and Akt3 mRNAs are expressed at much lower levels than Akt1 (Fig. 3H). Using isoform-specific antibodies, we detected strong Akt1 and Akt2 but much weaker Akt3 protein expression in MEFs (Fig. 3I). These data indicate that the isoform-specific roles of Akt1-3 in somatic cell reprogramming cannot be attributed simply to their relative expression levels in MEFs. Taken together, the functions of Akt1 and Akt3, but not Akt2, are essential to the proliferation and survival of reprogrammed cells, despite the fact that all three Akt isoforms can promote reprogramming and collaborate with LIF to stimulate Stat3 phosphorylation.

\section{Inhibiting GSK3 activity reverses the suppression of cell proliferation and reprogramming by Akt or mTOR inhibition} Both GSK3 and mTORC1 are downstream Akt targets. Suppressing mTORC1 activity with rapamycin has been shown to inhibit the generation of mouse iPSCs at the level of both ALPand Oct4-GFP-positive colony formation (He et al., 2012). This is very similar to our results with Akt1 and Akt3 knockdown (Fig. 3E,F). GSK3 is directly phosphorylated and inhibited by Akt (Cross et al., 1995), resulting in cell growth and proliferation. Because GSK3 $\beta$ also downregulates mTORC1 through activation of the negative regulator tuberous sclerosis 2 (Tsc2) (Inoki et al., 2006), we investigated whether inhibiting GSK3 would abrogate the effect of mTOR and Akt inhibition on reprogramming.

Two days after OKSM induction, the mTOR inhibitor rapamycin or the Akt inhibitor MK2206 were added to OGMEFs, with or without the addition of the GSK3 inhibitor CHIR99021 (Ying et al., 2008). On day 17, we observed obvious inhibition of cell proliferation by rapamycin or MK2206, compared to the DMSO-control (Fig. 4A). However, this was reversed by the addition of CHIR99021 (Fig. 4A). By 3 weeks, inhibition of Akt activity by MK2206 had greatly suppressed GFP-positive colony formation (Fig. 4B,C). A similar effect was observed by direct inhibition of mTOR using rapamycin (Fig. 4B,C), as was reported previously (He et al., 2012). However, in both cases, complete reprogramming was fully recovered with the addition of CHIR99021, as demonstrated by the number of GFP-positive colonies induced (Fig. 4B,C). These data strongly indicate that inhibition of GSK3 is an important component of the stimulation of cell proliferation and reprogramming by the Akt pathway.

To test whether blocking Akt activity affects the survival of reprogrammed cells, we analyzed the cell death 8 days after OKSM transduction, following treatment with MK2206, or 
A

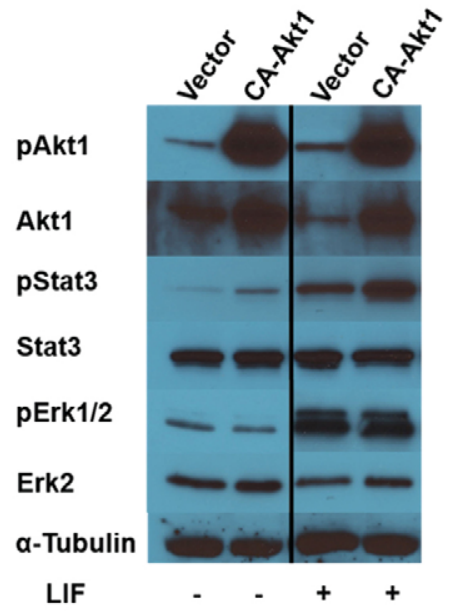

C

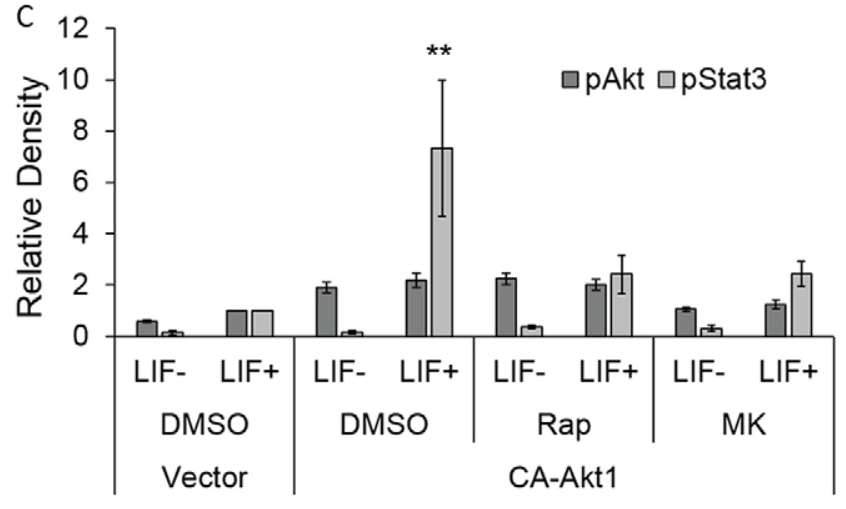

$\mathrm{E}$

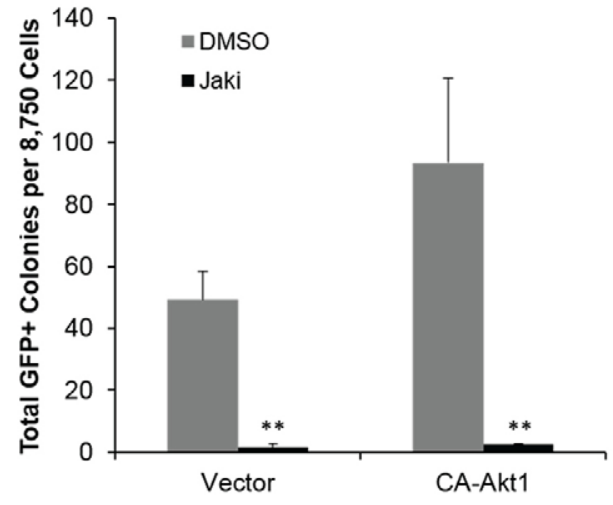

B
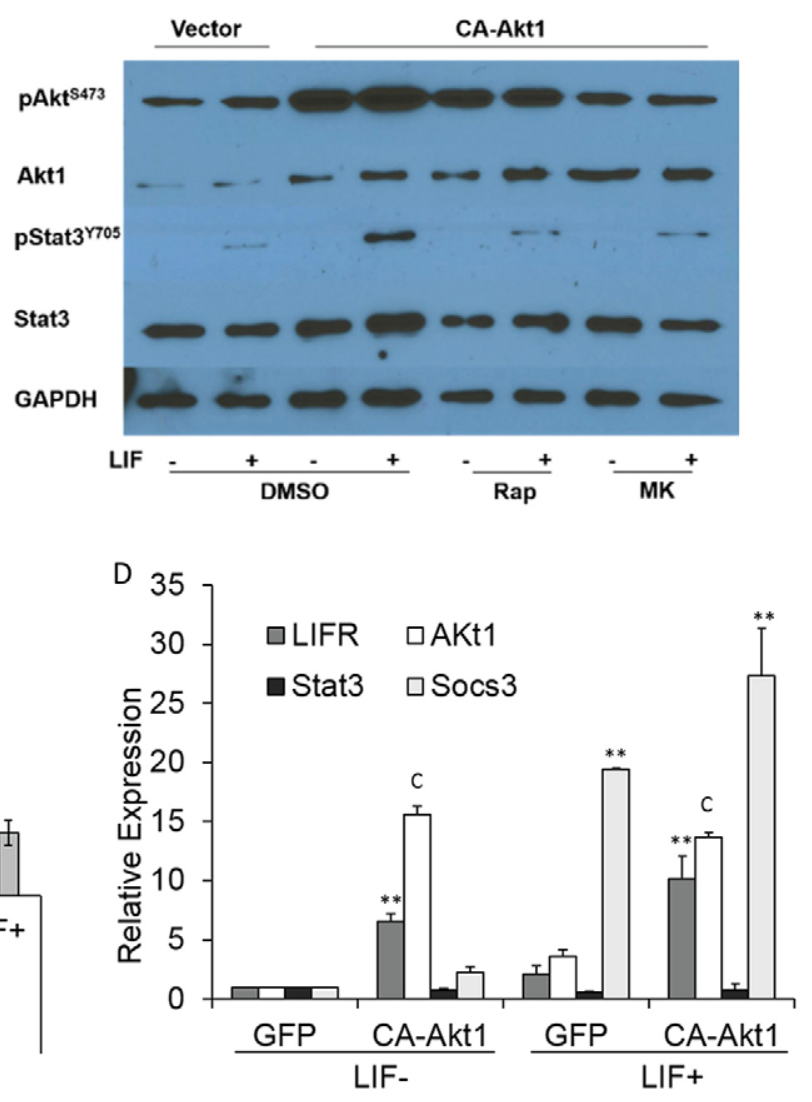

$\mathrm{F}$

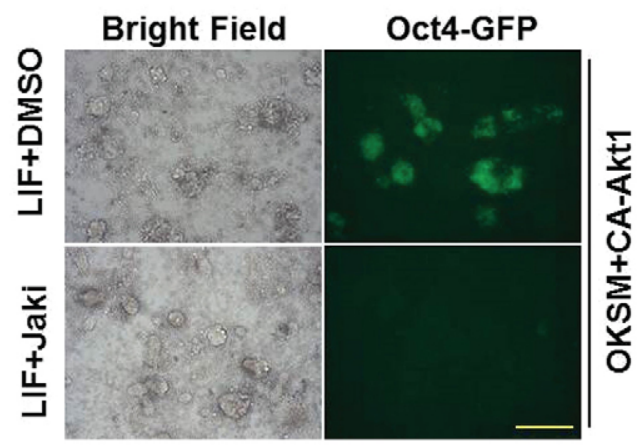

Fig. 2. Mechanism of Akt1 in promoting reprogramming. (A) Western blotting of total protein extracts from B6/129-MEFs transduced with either vector control or CA-Akt1, and with (+) or without (-) a 20 minute exposure to LIF. The specific proteins probed were Akt, Stat3, Erk1/2 and their respective phosphorylated forms. Tubulin was used as the loading control. Stimulation with LIF and CA-Akt1 co-stimulated Stat3 phosphorylation. (B) Western blotting of total protein extracts from B6/129-MEFs transduced with either vector control or CA-Akt1 and treated with DMSO, $5 \mu \mathrm{M}$ rapamycin (Rap) or $1 \mu \mathrm{M}$ MK2206 (MK), with (+) or without (-) LIF stimulation. The specific proteins probed were Akt, Stat3 and their respective phosphorylated forms. GAPDH was used as the loading control. Both mTOR and Akt inhibitors suppressed Stat3 phosphorylation. (C) Relative signal density of pStat3 and pAkt from western blots shown in B. The values were normalized with GAPDH and calculated relative to the levels in DMSO/Vector/LIF-treated cells. (D) Relative levels of mRNA for LIFR, Akt1, Stat3 and Socs3 in B6/129 MEFs transfected with retroviral GFP control or CA-Akt1, with (+) or without (-) 20 minutes of stimulation with LIF; c denotes combined endogenous and retroviral Akt1 expression. Values were normalized to GAPDH and expressed relative to the GFP control without stimulation with LIF. Compared to the GFP control without stimulation with LIF, the level of LIFR was significantly increased in CA-Akt1-transduced cells and highest in CA-Akt1 cells treated with LIF. As an indicator of Stat3 activation, the level of Socs 3 was significantly increased when cells had been treated with LIF and was highest in CAAkt1 cells treated with LIF, demonstrating a positive feedback effect of Akt1 on the LIF pathway. (E) Effect of Stat3 inhibition on formation of GFP-positive colonies induced by OKSM plus vector control or CA-Akt1. Transduced OG-MEFs were cultured in LIF-containing medium with either DMSO or $1 \mu$ M Jaki and observed 2 weeks after OKSM transduction. Inhibiting Stat3 completely blocked GFP-positive colony formation even in the presence of CA-Akt1.

(F) Representative ESC-like colonies induced in OG-MEFs and treated with either DMSO or Jaki as described in E. (Scale bar: $610 \mu \mathrm{m})$. Inhibiting Stat3 completely blocked GFP-positive expression but not colony formation. 
A

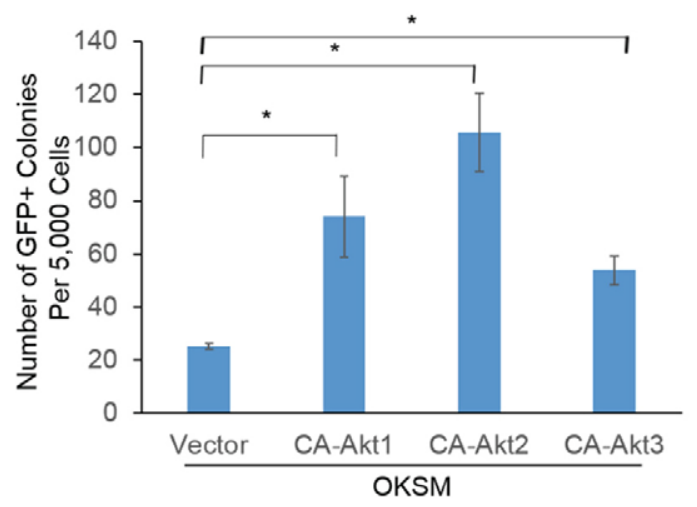

C

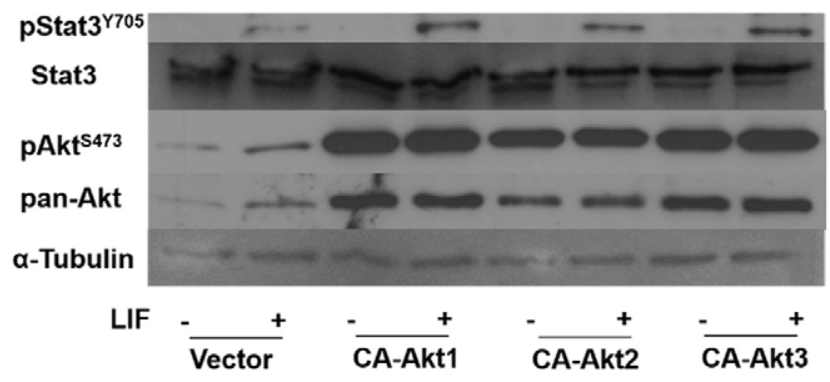

E
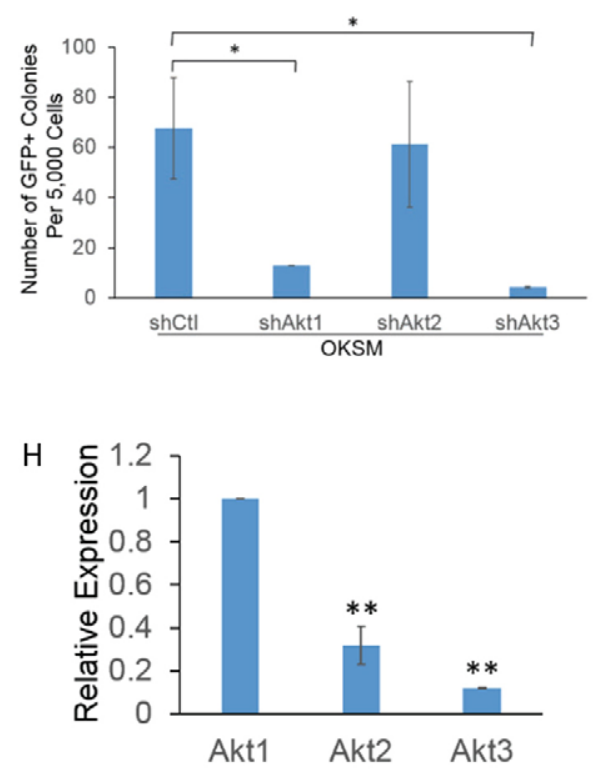

B
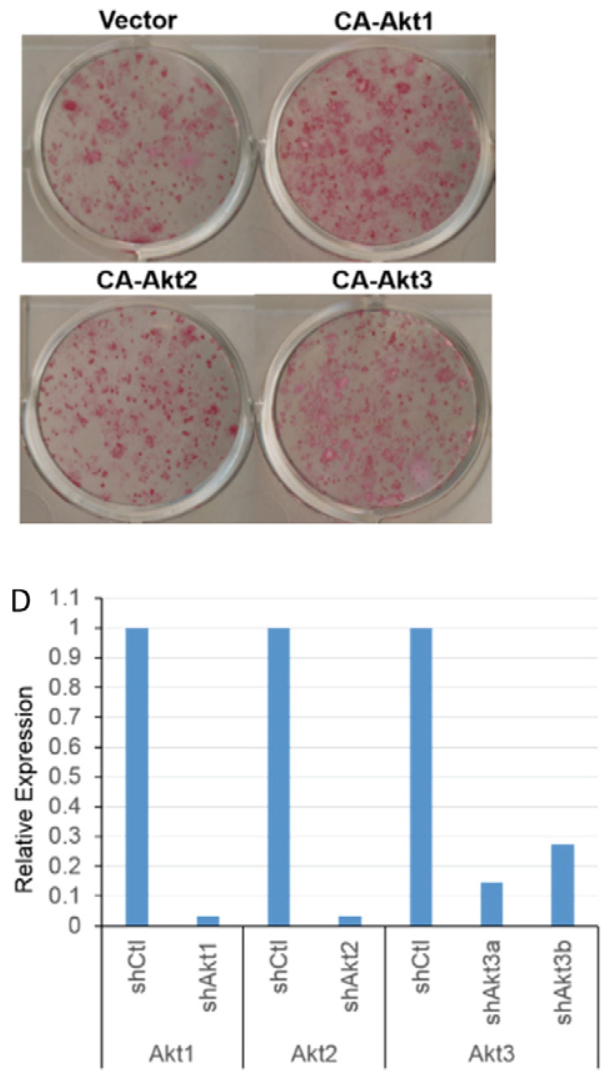

G

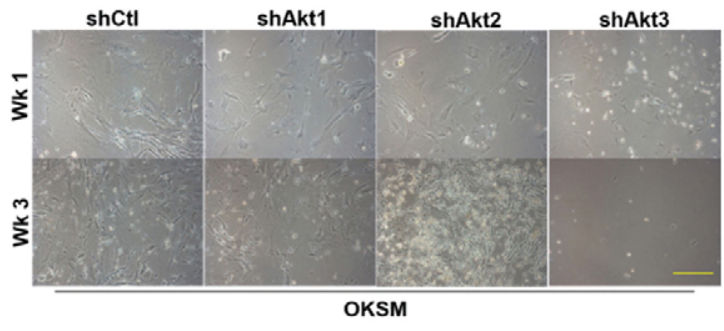

Fig. 3. See next page for legend.

MK2206 plus CHIR99021. On day 8 of reprogramming, treatment with MK2206 yielded a total number of cells less than $50 \%$ of the control, and this was completely reversed with the addition of CHIR99021 (Fig. 4D). Analysis of apoptosis revealed that treatment with MK2206 resulted in no obvious increase in cell death, with an early apoptotic cell population of $18.7 \%$ compared to $16.6 \%$ in the control (Fig. 4E). The addition of CHIR99021 reduced the early apoptotic cell population
(11.1\% in MK2206 plus CHIR99021 compared to $18.8 \%$ in MK2206 or $16.6 \%$ in control), which might partially but not fully explain the recovered total cell number on day 8 following CHIR99021 treatment (Fig. 4D,E). This indicates that after treatment with MK2206 $(1 \mu \mathrm{M})$, inhibition of Akt mainly reduces the proliferation rather than the survival of reprogrammed cells. Blocking GSK3 activity restores the total number of reprogrammed cells after MK2206 treatment, 
Fig. 3. All Akt isoforms promote efficient reprogramming and Stat3 phosphorylation, but only Akt1 and Akt3 are necessary for reprogramming. (A) The number of GFP-positive colonies induced by transduction of OG-MEFs with OKSM plus retroviral vector or CA-Akt1, CAAkt2, or CA-Akt3. The cells were cultured in reprogramming medium with LIF and observed 17 days after OKSM transduction. Similar to Akt1, both Akt2 and Akt3 also promote reprogramming. (B) Representative images of ALP staining of induced colonies 4 weeks after OKSM transduction as described in A. (C) Western blotting of total protein extracts from B6/129-MEFs transduced with either vector control, CA-Akt1, CA-Akt2 or CA-Akt3 and treated with (+) or without (-) LIF for 20 minutes. The specific proteins probed were pan-Akt, Stat3 and their respective phosphorylated forms. Similarly to Akt1, Akt2 and Akt3 acted collaboratively with the stimulation with LIF to stimulate Stat3 phosphorylation. (D) Relative expression levels of Akt1, Akt2 and Akt3 in B6/129 MEFs transduced with lentiviral shRNA constructs specific to each Akt isoform or control shRNA (shCtl). Gene expression values of duplicate sample preparations were normalized to GAPDH and expressed relative to the vector controls. (E) The number of GFP-positive colonies induced 3 weeks after OG-MEFs were transduced with OKSM plus lentiviral control (shCtl), shAkt1, shAkt2 or shAkt3, and cultured in reprogramming medium with LIF. Blocking Akt1 or Akt3, but not Akt2, suppressed the formation of GFP-positive colonies. (F) Representative images of ALP staining of the induced colonies 4 weeks after the OKSM transduction and RNA knockdown treatments described in E. Blocking Akt1 or Akt3, but not Akt2, suppressed the formation of colonies. (G) OG-MEFs transduced with shCtl, shAkt1, shAkt2 or shAkt3, 2 days after OKSM transduction and cultured in LIF-containing medium. Representative images of MEFs at 1 and 3 weeks after the initial OKSM transduction are shown (Scale bar: $250 \mu \mathrm{m}$ ). Blocking Akt1 or Akt3, but not Akt2, suppressed the proliferation of reprogrammed cells. $(H)$ Relative expression levels of Akt1, Akt2 and Akt3 mRNA in B6/129 MEFs normalized to GAPDH, with the value of Akt1 set as 1. (I) Western blotting of total protein extracts from B6/129 MEFs. The specific proteins probed were Akt1, Akt2 and Akt3. $\alpha$-tubulin was used as a loading control.

probably by improving both cell survival and the cell proliferation speed.

Esrrb has been identified as the essential downstream effector of GSK3 $\beta$ inhibition, and is responsible for stimulating ESC colony formation, preventing differentiation and promoting Nanog expression (Martello et al., 2012; van den Berg et al., 2008). However, how the GSK3 $\beta /$ Esrrb axis interacts with the Akt pathway is unclear. Interestingly, we found that, during the late reprogramming stage of OKSM transduction, treatment with MK2206 blocked expression of both Nanog and Esrrb, whereas addition of CHIR99021 restored expression of both factors (Fig. 4F). This indicates a contribution to reprogramming from the Akt/GSK3 pathway through Esrrb protein expression.

We, therefore, asked whether expression of Esrrb protein alone would rescue reprogramming halted by Akt inhibition. We transduced the Doxycycline (Dox)-inducible Esrrb lentiviral construct into OG-MEFs 2 days after OKSM transduction and, at day 4, added Dox with either MK2206 or DMSO to the reprogramming medium. Seven days after transduction, the

Table 2. Number (mean \pm s.d.) of total ESC-like colonies induced by OKSM plus retroviral vector, CA-Akt2, or CA-Akt3 in OG-MEFs 4 weeks after retroviral transduction. Starting cell number: $5000(n=2)$

\begin{tabular}{llll}
\hline \multirow{2}{*}{ Total ESC-like colonies } & \multicolumn{2}{l}{ OKSM } & \\
\cline { 2 - 4 } & Vector & CA-Akt2 & CA-Akt3 \\
\hline LIF+ & $246.7 \pm 20.3$ & $397.3 \pm 66.9$ & $432.8 \pm 106.3$ \\
\hline
\end{tabular}

LIF+, cells cultured with LIF. expression of Esrrb protein had begun to restore cell proliferation suppressed by MK2206 (Fig. 4G). Most significantly, by day 19 Esrrb had completely rescued reprogramming impeded by treatment with MK2206, as indicated by the full recovery of GFP-positive colony numbers (Fig. 4H,I). Thus, signaling from the GSK3/Esrrb axis plays an important role downstream of Akt to promote both cell proliferation and complete reprogramming.

\section{DISCUSSION}

Stat3 activation is required for the generation of completely reprogrammed iPSCs from somatic cells (Tang et al., 2012), and enhanced Stat3 activity further promotes reprogramming efficiency (Tang et al., 2012; van Oosten et al., 2012). LIF is a key component for reprogramming of somatic cells to naïve-state pluripotency, through stimulating Stat3. LIF also activates the PI3K/Akt pathway; but how this signaling pathway - which is responsible for many different biological processes - contributes to reprogramming remains unclear. During the preparation of this manuscript, a report was published on the role of Akt1 in mediating ES cell-expressed Ras signaling, that promoted reprogramming through phosphorylation and inhibition of Foxol (Yu et al., 2013). This revealed one layer of regulation by Akt1 in the reprogramming process. However, it is unclear whether inhibiting Foxol alone recapitulates the effect of Akt1 on reprogramming. Here, we show that all three Akt isoforms can promote reprogramming by maximizing Stat 3 activation in collaboration with LIF, rather than through another route which bypasses Stat 3 signaling. We discovered two pathways that are involved to maximize Stat3 activity through Akt signalling during reprogramming. One pathway involves activation of the mTOR Ser/Thr kinase that stimulates Stat3 activity, as previously described (Yokogami et al., 2000). The other pathway causes increased LIFR expression - a new effect of Akt revealed in this study, which establishes a positive feedback loop: LIF stimulation $\rightarrow$ increased levels of pAkt $\rightarrow$ LIFR expression $\rightarrow$ enhanced LIF signaling $\rightarrow$ enhanced pStat 3 levels. Whether these two routes operate in parallel or are both downstream of mTOR activation needs further clarification. Indeed, the study mentioned earlier also stated that enhanced ERas or Akt1 activation increased phosphorylation of Stat3 at Ser727 (Yu et al., 2013), which is the site phosphorylated by mTOR (Yokogami et al., 2000). Our results, thus, demonstrate a strong functional link between the Stat3 and Akt pathways during the process of complete reprogramming to pluripotency (Fig. 4J). Our finding that Stat3 activity is augmented by all three Akt isoforms also has important implications for cancer research because both Akt and Stat3 are frequently deregulated in and responsible for the development of various cancers (Gonzalez and McGraw, 2009; Yue and Turkson, 2009).

We found that the role of Akt2 in reprogramming is dispensable, contrary to that of Akt1 or Akt3, and that Akt2 is not essential for the proliferation of reprogrammed cells, a result probably not attributable to the lower expression of Akt2 in MEFs. In a previous study it was determined that, unlike Akt1 and Akt3, which target cell cytoplasm and nucleus, respectively, Akt2 mainly colocalizes with the mitochondria, even though comparison of Akt1 and Akt3 protein sequences did not reveal any striking differences that might be responsible for the different localization (Santi and Lee, 2010). Akt2 has been shown to regulate glucose metabolism, and a targeted Akt2 deletion in mice led to diabetic syndrome (Cho et al., 2001a; Garofalo et al., 

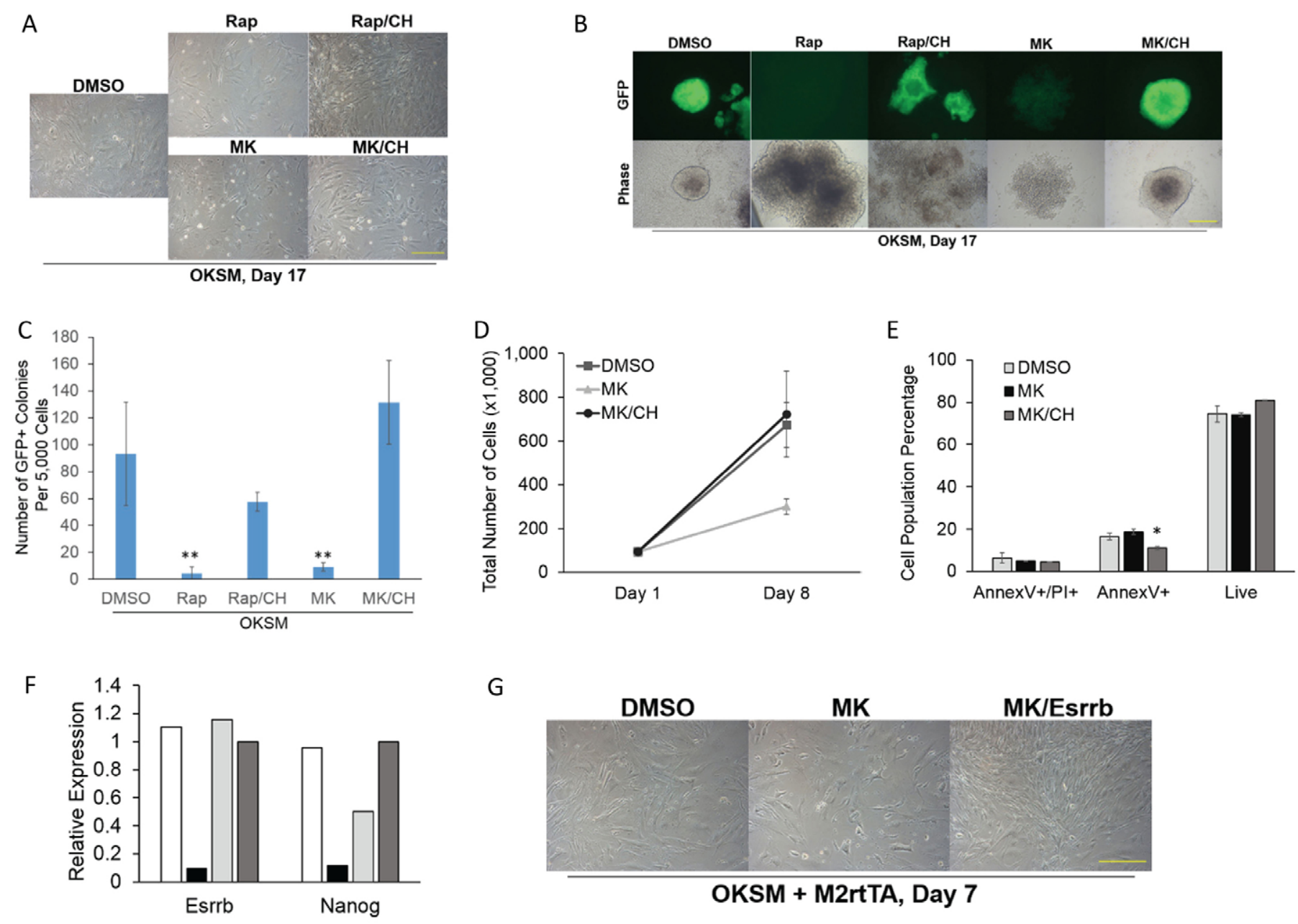

G

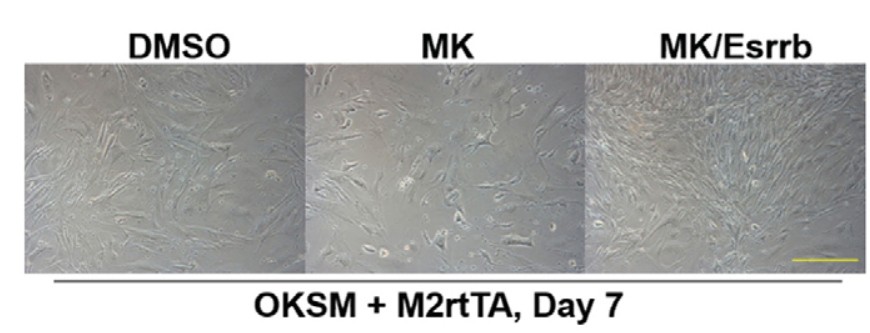

$\square D M S O=M K \square M K / C H \quad \square R 1$

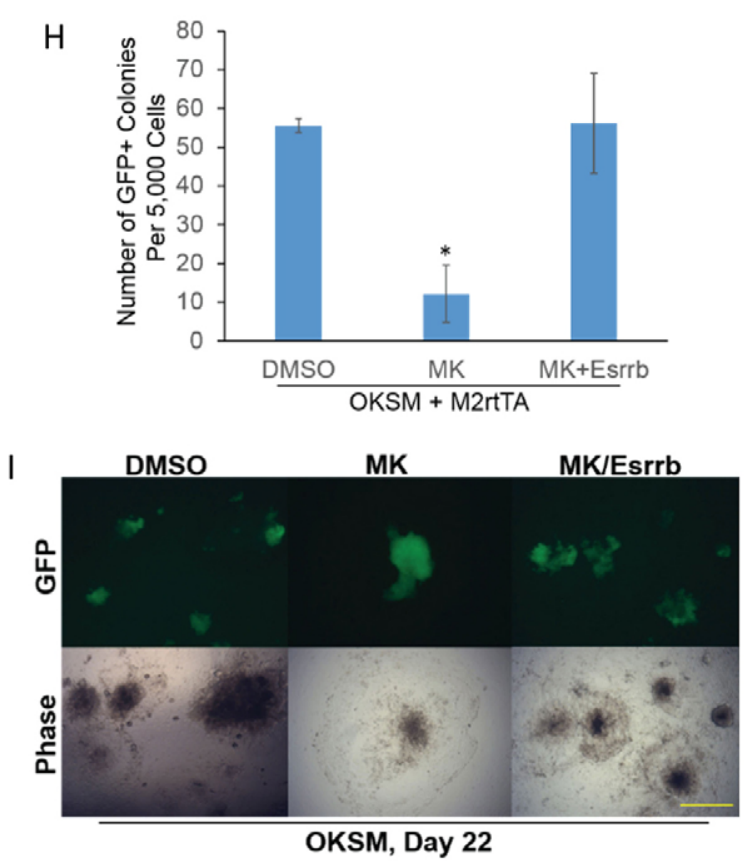

J

External Stimuli (e.g., LIF)
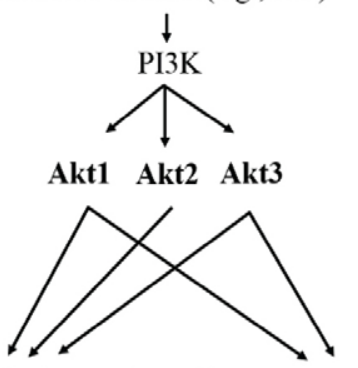

mTOR Activation/ Downstream Pathways LIFR Expression (mTOR, Foxol, GSK3/Esrrb?)

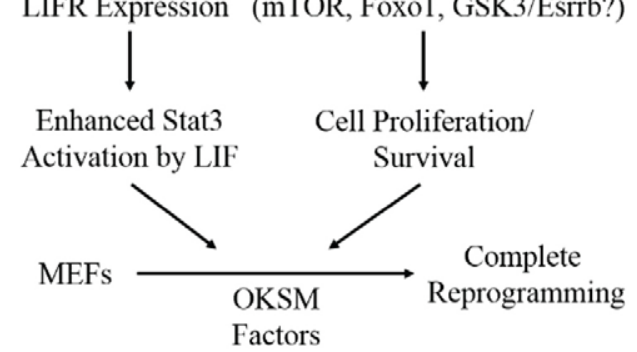

Fig. 4. See next page for legend. 
Fig. 4. Inhibiting GSK3 $\beta$ rescues cell proliferation and reprogramming halted by Akt or mTOR inhibition. (A) OG-MEFs were transduced with OKSM and treated with either DMSO, $1 \mu$ M MK2206MK (MK) or $5 \mu \mathrm{M}$ rapamycin (Rap), with or without $3 \mu \mathrm{M}$ CHIR99021 (CH), and cultured in LIFcontaining medium. Representative images of MEFs 17 days after the initial OKSM transduction are shown (Scale bar: $250 \mu \mathrm{m}$ ). Inhibiting mTOR or Akt suppressed the proliferation of reprogrammed cells; this can be reversed by CHIR. (B) GFP-positive colony formation by OG-MEFs transduced with OKSM and treated as in A. Representative colonies 17 days after the initial OKSM transduction are shown (Scale bar: $250 \mu \mathrm{m}$ ). (C) The number of GFPpositive colonies assayed 3 weeks after OG-MEF transduction with OKSM and treatment as described in A. Inhibiting mTOR or Akt suppressed the formation of GFP-positive colonies; this can be reversed by CHIR. (D) OGMEFs were transduced with OKSM on day 0 , replated on day 1 at $0.95 \times 10^{5}$ cells/well, and treated with either DMSO, MK or MK plus $3 \mu \mathrm{M} \mathrm{CHIR}(\mathrm{CH})$, and cultured in LIF-containing medium. Total cell numbers were determined 8 days after initial OKSM transduction. (E) Analysis of apoptosis in cells treated as described in D. Cells were incubated with Annexin V-FITC (AnnexV) and propidium iodide $(\mathrm{PI})$ for 30 minutes and analyzed by flow cytometry. Percentage of late apoptotic/necrotic (AnnexV+/PI+), early apoptotic (AnnexV+), and live cells (unstained) were shown. Addition of CHIR mildly suppresses apoptosis. (F) Relative levels of expression of Nanog and Esrrb mRNA in OKSM-transduced MEFs treated as described in A, 17 days after reprogramming. Values were normalized to GAPDH and calculated relative to levels found in R1 cells. Inhibiting Akt suppressed the activation of Esrrb and Nanog during reprogramming. (G) OG-MEFs transduced with OKSM plus M2rtTA, or OKSM plus M2rtTA and Dox-inducible Esrrb, treated with either DMSO or MK and cultured in LIF-containing medium with $1 \mu \mathrm{g} / \mathrm{ml}$ Dox. Representative images of reprogrammed cells 7 days after the initial OKSM transduction are shown (Scale bar: $250 \mu \mathrm{m}$ ). Expression of Esrrb reversed the suppression of cell proliferation caused by Akt inhibition. $(H)$ The number of GFP-positive colonies induced 19 days after OKSM induction as described in G. Expression of Esrrb restored the complete reprogramming that had been halted by Akt inhibition. (I) Representative GFP-positive colonies seen 22 days after OKSM induction as described in G. (J) Proposed model of Akt isoform-specific roles for successful reprogramming. Akt1, Akt2 and Akt3 enhance LIF-triggered Stat3 activation through stimulation of mTOR activation and LIFR expression; Akt1 and Akt 3 also collaboratively or differentially regulate their downstream targets to promote the proliferation and survival of reprogrammed cells. Both pathways contribute significantly to the successful generation of pluripotent cells.

2003; George et al., 2004). This role of Akt2 is, at least partially, mediated through regulating the intracellular translocation of glucose transporter type 4 (Glut4) - that promotes glucose influx - to the plasma membrane (Jiang et al., 2003; Katome et al., 2003). Enhanced activation of Akt 2 might, therefore, further promote cell proliferation and iPSC generation by stimulating glucose metabolism in reprogrammed cells, in addition to its function on pStat 3 stimulation.

mTOR activity has been found to be necessary for both somatic cell reprogramming (He et al., 2012) and for maintenance of pluripotency in ESCs (Betschinger et al., 2013). There are also multiple lines of evidence supporting crosstalk between the GSK3 $\beta$ and mTOR pathways. First, GSK $3 \beta$ phosphorylates and activates TSC2 to suppress mTORC1 activity (Inoki et al., 2006). Second, it was recently found that Esrrb, the downstream effector of GSK3 $\beta$ inhibition, plays a role downstream of mTOR in supporting ESC pluripotency (Betschinger et al., 2013; Martello et al., 2012). However, the consequence of this crosstalk for reprogramming was not determined. We found that, during reprogramming, inhibition of GSK3 $\beta$ completely rescues the cell proliferation and reprogramming blocked by $\mathrm{mTOR}$ or Akt inhibitors, which highlights the importance of inhibition of the GSK3 pathway in the Akt/mTOR-mediated reprogramming process.

Ectopic expression of Esrrb has been shown to induce iPSCs together with Oct4 and Sox2, through replacing Klf4 (Feng et al.,
2009). In ESCs, both Klf4 and Esrrb stimulate the expression of Nanog, a necessary component for maintenance of pluripotency (Niwa et al., 2009; van den Berg et al., 2008). Our results show that inhibition of Akt activity leads to suppressed Esrrb and Nanog expression in reprogrammed cells, and that this suppression can be recovered by CHIR99021 treatment. We further demonstrate that the cell proliferation and complete reprogramming that is halted by Akt inhibition can be reversed by expressing Esrrb protein. Our results, thus, strongly indicate that Esrrb serves as a main downstream effector of Akt/GSK3 $\beta$ signaling, and promotes somatic cell reprogramming through enhancing cell proliferation and pluripotency establishment.

We found that both Akt1 and Akt3 are needed for successful reprogramming. The non-overlapping functions of Akt1 and Akt3 in reprogramming may reflect the differential subcellular localization of these two proteins (Santi and Lee, 2010). We also discovered that the promotion of reprogramming is tightly correlated with the role of both isoforms in the maintenance of cell proliferation. Because our results show that knockdown of either Akt1 or Akt3 resembles the effect on reprogramming by using mTOCR1 inhibition through rapamycin (He et al., 2012) or a constitutively active form of Foxol ( $\mathrm{Yu}$ et al., 2013), we suggest that Akt1 and Akt3 regulate - collaboratively or differentially - their downstream targets, such as mTOR and Foxo1 or the GSK3 $\beta /$ Esrrb axis, in supporting cell proliferation and survival during reprogramming (Fig. 4J).

Our results described herein illustrate a key role of Akt in coordinating somatic cell reprogramming by supporting the proliferation of reprogrammed cells through the Akt isoforms 1 and 3, co-stimulating the phosphorylation of Stat3 with LIF to promote pluripotency establishment, and regulating the GSK3 pathways to promote both cell proliferation and reprogramming via Esrrb. The results obtained here, not only significantly contribute to our understanding of the mechanism for somatic cell reprogramming but also have important implications for cancer research.

\section{MATERIALS AND METHODS}

\section{Chemicals and protein expression constructs}

Jak inhibitor I (Jaki) and Doxycycline (Dox) were purchased from EMD Millipore. Rapamycin, MK2206 and GSK3 $\beta$ inhibitor CHIR99021 were obtained from SelleckChem. The constitutively active mouse Akt isoforms 1, 2 and 3 (CA-Akt1, CA-Akt2 and CA-Akt3) comprising the N-terminal myriostoylation sequence MGSSKSKPK, were amplified by RT-PCR from mouse cDNAs by using specific primers. The PCR products were then subcloned into pMXs- or pMCs-vectors (Cell Biolabs). The vectors for pMXs-Oct4, Klf4, Sox2 and c-Myc (Takahashi and Yamanaka, 2006), pLKO.1-puro, pLKO.1-scramble shRNA control, FUW-M2rtTA and FUW-TetO-Esrrb (Buganim et al., 2012), retroviral and lentiviral packaging constructs pUMVC, pCMVVSV-G and psPAX2 (Stewart et al., 2003) were all obtained from Addgene. pLKO.1-shRNA constructs against mouse Akt1 and Akt2 were purchased from Sigma. DNA oligonucleotides against the mouse Akt3 cDNA (shAkt3a and shAkt3b) were subcloned into pLKO.1-puro vector. The human embryonic kidney cell line 293T (HEK 293T) for viral packaging was purchased from Invitrogen. The primer sequences for CAAkt1, CA-Akt2 and CA-Akt3 and Akt3 shRNA cloning are listed in supplementary material Table S1. All DNA subcloning was performed using standard restriction-enzyme digestion or the Infusion PCR Cloning Kit (Clontech) and expression constructs were verified by DNA sequencing.

\section{Cell culture, viral preparation and reprogramming assay}

OG-MEFs, as well as MEFs from strain B6/129 and CD1 MEF feeder cells were generated from E13.5 embryos as described previously (Tang 
et al., 2011). MEFs passaged up to four times were used for reprogramming. pMXs, pMCs or pLKO.1 constructs, together with packaging vectors pUMVC (for retrovirus), psPAX2 (for lentivirus) and pCMV-VSV-G plasmids, were co-transfected into $293 \mathrm{~T}$ cells according to protocols provided by Addgene. The assay for iPSC induction from OG-MEFs by using viral OKSM and reprogramming medium was conducted as described previously (Tang et al., 2011). Retroviral CAAkt1, CA-Akt2 and CA-Akt3, Dox-inducible M2rtTA that expresses reverse tetracycline transactivator, and TetO-Esrrb constructs were transduced to reprogrammed cells 2 days after OKSM transduction (day 0). All chemicals including DMSO, rapamycin, MK2206 and Dox were added on day 3, and GFP-positive colonies were scored 2-3 weeks after OKSM transduction by using a Nikon fluorescence microscope.

\section{Embryoid body (EB) formation}

Established iPSC cell lines that had been passaged six times were plated on CD1 MEF feeder cells. Two days later, the cells were trypsinized and re-plated on the original plate for 2 hours to allow MEFs to attach. The iPSCs remaining in the medium were transferred to plastic Petri dishes containing DMEM plus 10\% FBS (Invitrogen) without LIF. After 1 week of differentiation, EBs formed and were transferred to $0.1 \%$ gelatincoated cell culture dishes (Invitrogen). The cells were allowed to reattach and continue to differentiate for 7 days before proceeding with RNA extraction and real-time qRT-PCR or immunostaining, as described below.

\section{Immunostaining and alkaline phosphatase staining}

Alkaline phosphatase (ALP) staining was performed using a Vector Red Alkaline Phosphate Substrate Kit I (Vector Laboratories, Burlingame, CA) according to the manufacturer's instruction. For immunostaining, cells were grown on $12 \mathrm{~mm}$ glass coverslips (Fisher Scientific) in 6-well plates seeded with CD1 MEFs as feeders. Cells were fixed in $4 \%$ paraformaldehyde with $1 \%$ sucrose in PBS for $15 \mathrm{~min}$ at room temperature. The cell membranes were permeabilized with $0.5 \%$ Triton $\mathrm{X}-100$ in PBS plus TWEEN 20 (PBS-T), then incubated for $2 \mathrm{hrs}$ at $37^{\circ} \mathrm{C}$ in $5 \%$ donkey or goat serum with mouse anti-SSEA-1 IgM (1:100), rabbit anti-Sox2 IgG (1:100) or rabbit anti-Nanog IgG (1:100) (all from Millipore), or rabbit anti-Oct4 antibody (1:100, Santa Cruz), washed in PBS-T and then incubated with Alexa-Fluor-594-conjugated donkey antirabbit or goat anti-mouse secondary antibodies (1:500, Invitrogen). After the washes, cells were counterstained with DAPI and mounted under coverslips. Fluorescence images were taken using a Nikon fluorescence microscope.

\section{Western blotting}

MEFs were transduced with retroviral-vector CA-Akt1, CA-Akt2 or CAAkt 3 and cultured in medium containing 10\% FBS for 4 days. The cells were then stimulated with either $1000 \mathrm{U} / \mathrm{ml} \mathrm{LIF}$ or PBS for 20 mins. Total cellular proteins were extracted using RIPA buffer (Thermo Scientific, Pittsburg, PA) supplemented with $1 \times$ proteinase and phosphatase inhibitors (Thermo Scientific). Proteins were quantified by using a BCA-Quantification kit (Thermo Scientific) and subjected to $10 \%$ SDS-PAGE gel electrophoresis using a BioRad mini-gel system before being transferred to PVDF membranes.

The blotted membranes were blocked with $5 \%$ non-fat dry milk in TBS plus TWEEN 20 (TBS-T) and incubated with primary antibodies at $4{ }^{\circ} \mathrm{C}$ overnight. The antibodies used were as follows: anti-Akt1 (1:5,000, Millipore), anti-pAkt (1:5000, Cell Signaling, Danvers, MA), anti-Stat3 (1:2500, BD, Franklin Lakes, NJ), anti-pStat3 (1:5000, Cell Signaling), anti-Erk2 (1:1000, BD), anti-pErk1/2 (1:2000, Millipore), and anti- $\alpha-$ Tubulin (1:2000, Sigma). Other Akt isoforms and pan-Akt were blotted by using Akt isoform antibody sampler kit from Cell Signaling. Membranes were then washed and blotted with horseradish peroxidase (HRP)conjugated goat anti-mouse or goat anti-rabbit secondary antibodies (1:5000, Santa Cruz, Santa Crutz, CA). Chemiluminescence was detected by using the Pierce ECL Western-Blot Substrate (Thermo Scientific) and $\mathrm{X}$-ray film exposure. Developed films were scanned and quantified for signal density by using ImageJ software (Schneider et al., 2012).

\section{Quantitative real-time reverse transcription (RT)-PCR analysis}

Total RNA was extracted using the RNeasy Extraction kit (Qiagen), reverse transcribed using a SuperScript III Reverse Transcription Kit (Invitrogen) and amplified with specific primers (supplementary material Table S2). Real-time qRT-PCR was performed by using the SYBR Green PCR Master Mix (ABI) and the ABI 7500 Fast instrument. Data were by analyzed using the 7500 software version 2.0 .2 provided with the instrument. All values were normalized by using GAPDH as the internal control and relative mRNA expressions were quantified by using the R1ESC cell line (R1) as the reference.

\section{Apoptosis assay}

Cells on day 8 of reprogramming were trypsinized and labeled by using an Annexin V-FITC apoptosis detection kit (Sigma). Briefly, after trypsinization, cells from each condition were resuspended in $0.5 \mathrm{ml}$ $1 \times$ binding buffer and incubated with $0.125 \mathrm{ug} / \mathrm{ml}$ Annexin V-FITC (Annex) and $2 \mathrm{ug} / \mathrm{ml}$ propidium iodide (PI) for 30 minutes. Cells were then analyzed with a BD FACSCalibur flow cytometer with fluorescence excitation at $488 \mathrm{~nm}$. Annexin V single-positive and Annexin/PI doublepositive cell gates were established based on populations seen in singly labeled samples. The cell populations were analyzed using FlowJo software.

\section{Statistical analysis}

Data were analyzed using One-Way ANOVA with Tukey's multiple comparisons or the Student's $t$-test. All experiments were performed at least twice $(n \geq 2)$. Figures were presented as mean \pm standard deviation (s.d.). ${ }^{*} P<0.05$ or $* * P<0.01$ were considered statistically significant.

\section{Competing interests}

The authors declare no competing interests.

\section{Author contributions}

Y.T.: Conception and design, collection and/or assembly of data, data analysis and interpretation, and manuscript writing. Z.J., Y.L., X.Z., L.W.: Collection and analysis of data. C.N.: Collection and analysis of data, manuscript writing. X.C.T. Conception and design, data interpretation, financial and administrative support, and manuscript writing.

\section{Funding}

This project was supported by the United States Department of Agriculture [grant number: 1265-31000-091-02S].

\section{Supplementary material}

Supplementary material available online at

http://jcs.biologists.org/lookup/suppl/doi:10.1242/jcs.150029/-/DC1

\section{References}

Alessi, D. R., James, S. R., Downes, C. P., Holmes, A. B., Gaffney, P. R., Reese, C. B. and Cohen, P. (1997). Characterization of a 3-phosphoinositidedependent protein kinase which phosphorylates and activates protein kinase Balpha. Curr. Biol. 7, 261-269.

Betschinger, J., Nichols, J., Dietmann, S., Corrin, P. D., Paddison, P. J. and Smith, A. (2013). Exit from pluripotency is gated by intracellular redistribution of the bHLH transcription factor Tfe3. Cell 153, 335-347.

Buganim, Y., Faddah, D. A., Cheng, A. W., Itskovich, E., Markoulaki, S., Ganz, K., Klemm, S. L., van Oudenaarden, A. and Jaenisch, R. (2012). Single-cell expression analyses during cellular reprogramming reveal an early stochastic and a late hierarchic phase. Cell 150, 1209-1222.

Burdon, T., Stracey, C., Chambers, I., Nichols, J. and Smith, A. (1999). Suppression of SHP-2 and ERK signalling promotes self-renewal of mouse embryonic stem cells. Dev. Biol. 210, 30-43.

Burdon, T., Smith, A. and Savatier, P. (2002). Signalling, cell cycle and pluripotency in embryonic stem cells. Trends Cell Biol. 12, 432-438.

Chen, W. S., Xu, P. Z., Gottlob, K., Chen, M. L., Sokol, K., Shiyanova, T., Roninson, I., Weng, W., Suzuki, R., Tobe, K. et al. (2001). Growth retardation and increased apoptosis in mice with homozygous disruption of the Akt1 gene. Genes Dev. 15, 2203-2208.

Chin, Y. R. and Toker, A. (2011). Akt isoform-specific signaling in breast cancer: uncovering an anti-migratory role for palladin. Cell Adh. Migr. 5, 211-214.

Cho, H., Mu, J., Kim, J. K., Thorvaldsen, J. L., Chu, Q., Crenshaw, E. B., III, Kaestner, K. H., Bartolomei, M. S., Shulman, G. I. and Birnbaum, M. J. (2001a). Insulin resistance and a diabetes mellitus-like syndrome in mice lacking the protein kinase Akt2 (PKB beta). Science 292, 1728-1731. 
Cho, H., Thorvaldsen, J. L., Chu, Q., Feng, F. and Birnbaum, M. J. (2001b) Akt1/PKBalpha is required for normal growth but dispensable for maintenance of glucose homeostasis in mice. J. Biol. Chem. 276, 38349-38352.

Cross, D. A., Alessi, D. R., Cohen, P., Andjelkovich, M. and Hemmings, B. A. (1995). Inhibition of glycogen synthase kinase-3 by insulin mediated by protein kinase B. Nature 378, 785-789.

Feng, B., Jiang, J., Kraus, P., Ng, J. H., Heng, J. C., Chan, Y. S., Yaw, L. P., Zhang, W., Loh, Y. H., Han, J. et al. (2009). Reprogramming of fibroblasts into induced pluripotent stem cells with orphan nuclear receptor Esrrb. Nat. Cell Biol. 11, 197-203.

Gabbert, H., Wagner, R., Moll, R. and Gerharz, C. D. (1985). Tumor dedifferentiation: an important step in tumor invasion. Clin. Exp. Metastasis 3, 257-279.

Garofalo, R. S., Orena, S. J., Rafidi, K., Torchia, A. J., Stock, J. L., Hildebrandt, A. L., Coskran, T., Black, S. C., Brees, D. J., Wicks, J. R. et al. (2003). Severe diabetes, age-dependent loss of adipose tissue, and mild growth deficiency in mice lacking Akt2/PKB beta. J. Clin. Invest. 112, 197-208.

George, S., Rochford, J. J., Wolfrum, C., Gray, S. L., Schinner, S., Wilson, J. C., Soos, M. A., Murgatroyd, P. R., Williams, R. M., Acerini, C. L. et al. (2004). A family with severe insulin resistance and diabetes due to a mutation in AKT2. Science 304, 1325-1328.

Gonzalez, E. and McGraw, T. E. (2009). The Akt kinases: isoform specificity in metabolism and cancer. Cell Cycle 8, 2502-2508.

He, J., Kang, L., Wu, T., Zhang, J., Wang, H., Gao, H., Zhang, Y., Huang, B., Liu, W., Kou, Z. et al. (2012). An elaborate regulation of Mammalian target of rapamycin activity is required for somatic cell reprogramming induced by defined transcription factors. Stem Cells Dev. 21, 2630-2641.

Heitman, J., Movva, N. R. and Hall, M. N. (1991). Targets for cell cycle arrest by the immunosuppressant rapamycin in yeast. Science 253, 905-909.

Herreros-Villanueva, M., Zhang, J. S., Koenig, A., Abel, E. V., Smyrk, T. C., Bamlet, W. R., de Narvajas, A. A., Gomez, T. S., Simeone, D. M., Bujanda, L. et al. (2013). SOX2 promotes dedifferentiation and imparts stem cell-like features to pancreatic cancer cells. Oncogenesis 2, e61.

Hirai, H., Sootome, H., Nakatsuru, Y., Miyama, K., Taguchi, S., Tsujioka, K., Ueno, Y., Hatch, H., Majumder, P. K., Pan, B. S. et al. (2010). MK-2206, an allosteric Akt inhibitor, enhances antitumor efficacy by standard chemotherapeutic agents or molecular targeted drugs in vitro and in vivo. Mol. Cancer Ther. 9, 1956-1967.

Inoki, K., Ouyang, H., Zhu, T., Lindvall, C., Wang, Y., Zhang, X., Yang, Q., Bennett, C., Harada, Y., Stankunas, K. et al. (2006). TSC2 integrates Wnt and energy signals via a coordinated phosphorylation by AMPK and GSK3 to regulate cell growth. Cell 126, 955-968.

Jiang, Z. Y., Zhou, Q. L., Coleman, K. A., Chouinard, M., Boese, Q. and Czech, M. P. (2003). Insulin signaling through Akt/protein kinase B analyzed by small interfering RNA-mediated gene silencing. Proc. Natl. Acad. Sci. USA 100, 7569-7574.

Katome, T., Obata, T., Matsushima, R., Masuyama, N., Cantley, L. C., Gotoh, Y., Kishi, K., Shiota, H. and Ebina, Y. (2003). Use of RNA interference-mediated gene silencing and adenoviral overexpression to elucidate the roles of AKT/ protein kinase B isoforms in insulin actions. J. Biol. Chem. 278, 28312-28323.

Kimura, T., Tomooka, M., Yamano, N., Murayama, K., Matoba, S., Umehara, H., Kanai, Y. and Nakano, T. (2008). AKT signaling promotes derivation of embryonic germ cells from primordial germ cells. Development 135, 869-879.

Laplante, M. and Sabatini, D. M. (2012). mTOR signaling in growth control and disease. Cell 149, 274-293

Lee, M. W., Kim, D. S., Lee, J. H., Lee, B. S., Lee, S. H., Jung, H. L., Sung, K. W., Kim, H. T., Yoo, K. H. and Koo, H. H. (2011). Roles of AKT1 and AKT2 in non-small cell lung cancer cell survival, growth, and migration. Cancer Sci. 102, 1822-1828.

Ma, J., Meng, Y., Kwiatkowski, D. J., Chen, X., Peng, H., Sun, Q., Zha, X. Wang, F., Wang, Y., Jing, Y. et al. (2010). Mammalian target of rapamycin regulates murine and human cell differentiation through STAT3/p63/Jagged/ Notch cascade. J. Clin. Invest. 120, 103-114.

Manning, B. D. and Cantley, L. C. (2007). AKT/PKB signaling: navigating downstream. Cell 129, 1261-1274.

Martello, G., Sugimoto, T., Diamanti, E., Joshi, A., Hannah, R., Ohtsuka, S., Göttgens, B., Niwa, H. and Smith, A. (2012). Esrrb is a pivotal target of the Gsk3/ Tcf3 axis regulating embryonic stem cell self-renewal. Cell Stem Cell 11, 491-504.

Nakamura, T., Inoue, K., Ogawa, S., Umehara, H., Ogonuki, N., Miki, H. Kimura, T., Ogura, A. and Nakano, T. (2008). Effects of Akt signaling on nuclear reprogramming. Genes Cells 13, 1269-1277.

Nesterov, A., Lu, X., Johnson, M., Miller, G. J., Ivashchenko, Y. and Kraft, A. S. (2001). Elevated AKT activity protects the prostate cancer cell line LNCaP from TRAIL-induced apoptosis. J. Biol. Chem. 276, 10767-10774.

Niwa, H., Burdon, T., Chambers, I. and Smith, A. (1998). Self-renewal of pluripotent embryonic stem cells is mediated via activation of STAT3. Genes Dev. 12, 2048-2060.

Niwa, H., Ogawa, K., Shimosato, D. and Adachi, K. (2009). A parallel circuit of LIF signalling pathways maintains pluripotency of mouse ES cells. Nature 460, 118-122.

Paling, N. R., Wheadon, H., Bone, H. K. and Welham, M. J. (2004). Regulation of embryonic stem cell self-renewal by phosphoinositide 3-kinase-dependent signaling. J. Biol. Chem. 279, 48063-48070.

Rathjen, P. D., Toth, S., Willis, A., Heath, J. K. and Smith, A. G. (1990). Differentiation inhibiting activity is produced in matrix-associated and diffusible forms that are generated by alternate promoter usage. Cell 62, 1105-1114

Saji, M., Narahara, K., McCarty, S. K., Vasko, V. V., La Perle, K. M., Porter, K., Jarjoura, D., Lu, C., Cheng, S. Y. and Ringel, M. D. (2011). Akt1 deficiency delays tumor progression, vascular invasion, and distant metastasis in a murine model of thyroid cancer. Oncogene 30, 4307-4315.
Santi, S. A. and Lee, H. (2010). The Akt isoforms are present at distinct subcellular locations. Am. J. Physiol. 298, C580-C591.

Sarbassov, D. D., Guertin, D. A., Ali, S. M. and Sabatini, D. M. (2005). Phosphorylation and regulation of Akt/PKB by the rictor-mTOR complex. Science 307, 1098-1101.

Schneider, C. A., Rasband, W. S. and Eliceiri, K. W. (2012). NIH Image to ImageJ: 25 years of image analysis. Nat. Methods 9, 671-675.

Schwitalla, S., Fingerle, A. A., Cammareri, P., Nebelsiek, T., Göktuna, S. I., Ziegler, P. K., Canli, O., Heijmans, J., Huels, D. J., Moreaux, G. et al. (2013). Intestinal tumorigenesis initiated by dedifferentiation and acquisition of stemcell-like properties. Cell 152, 25-38

Silva, J., Nichols, J., Theunissen, T. W., Guo, G., van Oosten, A. L., Barrandon, O., Wray, J., Yamanaka, S., Chambers, I. and Smith, A. (2009). Nanog is the gateway to the pluripotent ground state. Cell 138, 722-737.

Singh, A. M., Reynolds, D., Cliff, T., Ohtsuka, S., Mattheyses, A. L., Sun, Y., Menendez, L., Kulik, M. and Dalton, S. (2012). Signaling network crosstalk in human pluripotent cells: a Smad2/3-regulated switch that controls the balance between self-renewal and differentiation. Cell Stem Cell 10, 312326.

Smith, A. G., Heath, J. K., Donaldson, D. D., Wong, G. G., Moreau, J., Stahl, M. and Rogers, D. (1988). Inhibition of pluripotential embryonic stem cell differentiation by purified polypeptides. Nature 336, 688-690.

Stahl, J. M., Sharma, A., Cheung, M., Zimmerman, M., Cheng, J. Q., Bosenberg, M. W., Kester, M., Sandirasegarane, L. and Robertson, G. P. (2004). Deregulated Akt3 activity promotes development of malignant melanoma. Cancer Res. 64, 7002-7010.

Stewart, C. L., Kaspar, P., Brunet, L. J., Bhatt, H., Gadi, I., Köntgen, F. and Abbondanzo, S. J. (1992). Blastocyst implantation depends on maternal expression of leukaemia inhibitory factor. Nature 359, 76-79.

Stewart, S. A., Dykxhoorn, D. M., Palliser, D., Mizuno, H., Yu, E. Y., An, D. S., Sabatini, D. M., Chen, I. S., Hahn, W. C., Sharp, P. A. et al. (2003). Lentivirusdelivered stable gene silencing by RNAi in primary cells. RNA 9, 493-501.

Takahashi, K. and Yamanaka, S. (2006). Induction of pluripotent stem cells from mouse embryonic and adult fibroblast cultures by defined factors. Cell 126, 663-676.

Tang, Y., Lin, C. J. and Tian, X. C. (2011). Functionality and transduction condition evaluation of recombinant KIf4 for improved reprogramming of iPS cells. Cell Reprogram 13, 99-112.

Tang, Y., Luo, Y., Jiang, Z., Ma, Y., Lin, C. J., Kim, C., Carter, M. G., Amano, T., Park, J., Kish, S. et al. (2012). Jak/Stat3 signaling promotes somatic cell reprogramming by epigenetic regulation. Stem Cells 30, 2645-2656.

Tschopp, O., Yang, Z. Z., Brodbeck, D., Dummler, B. A., HemmingsMieszczak, M., Watanabe, T., Michaelis, T., Frahm, J. and Hemmings, B. A. (2005). Essential role of protein kinase B gamma (PKB gamma/Akt3) in postnatal brain development but not in glucose homeostasis. Development 132, 2943-2954.

van den Berg, D. L., Zhang, W., Yates, A., Engelen, E., Takacs, K., Bezstarosti, K., Demmers, J., Chambers, I. and Poot, R. A. (2008). Estrogen-related receptor beta interacts with Oct4 to positively regulate Nanog gene expression. Mol. Cell. Biol. 28, 5986-5995.

van Oosten, A. L., Costa, Y, Smith, A and Silva, J. C. (2012). JAK/STAT3 signalling is sufficient and dominant over antagonistic cues for the establishment of naive pluripotency. Nat. Commun. 3, 817.

Vanhaesebroeck, B. and Alessi, D. R. (2000). The PI3K-PDK1 connection: more than just a road to PKB. Biochem. J. 346, 561-576.

Watanabe, S., Umehara, H., Murayama, K., Okabe, M., Kimura, T. and Nakano, T. (2006). Activation of Akt signaling is sufficient to maintain pluripotency in mouse and primate embryonic stem cells. Oncogene 25, 2697-2707.

Williams, R. L., Hilton, D. J., Pease, S., Willson, T. A., Stewart, C. L., Gearing, D. P., Wagner, E. F., Metcalf, D., Nicola, N. A. and Gough, N. M. (1988). Myeloid leukaemia inhibitory factor maintains the developmental potential of embryonic stem cells. Nature 336, 684-687.

Yang, Z. Z., Tschopp, O., Baudry, A., Dümmler, B., Hynx, D. and Hemmings, B. A. (2004). Physiological functions of protein kinase B/Akt. Biochem. Soc. Trans. 32, 350-354

Ying, Q. L., Wray, J., Nichols, J., Batlle-Morera, L., Doble, B., Woodgett, J., Cohen, P. and Smith, A. (2008). The ground state of embryonic stem cell selfrenewal. Nature 453, 519-523.

Yokogami, K., Wakisaka, S., Avruch, J. and Reeves, S. A. (2000). Serine phosphorylation and maximal activation of STAT3 during CNTF signaling is mediated by the rapamycin target mTOR. Curr. Biol. 10, 47-50.

Yu, J., Vodyanik, M. A., Smuga-Otto, K., Antosiewicz-Bourget, J., Frane, J. L., Tian, S., Nie, J., Jonsdottir, G. A., Ruotti, V., Stewart, R. et al. (2007). Induced pluripotent stem cell lines derived from human somatic cells. Science 318, 1917-1920.

Yu, Y., Liang, D., Tian, Q., Chen, X., Jiang, B., Chou, B. K., Hu, P., Cheng, L., Gao, P., Li, J. et al. (2013). Stimulation of Somatic Cell Reprogramming by Eras-Akt-Foxo1 Signaling Axis. Stem Cells

Yue, P. and Turkson, J. (2009). Targeting STAT3 in cancer: how successful are we? Expert Opin. Investig. Drugs 18, 45-56.

Zhang, B., Gu, F., She, C., Guo, H., Li, W., Niu, R., Fu, L., Zhang, N. and Ma, Y. (2009). Reduction of Akt2 inhibits migration and invasion of glioma cells. Int. J. Cancer 125, 585-595.

Zhang, H., Wu, H., Zheng, J., Yu, P., Xu, L., Jiang, P., Gao, J., Wang, H. and Zhang, Y. (2013). Transforming growth factor $\beta 1$ signal is crucial for dedifferentiation of cancer cells to cancer stem cells in osteosarcoma. Stem Cells 31, 433-446. 